\title{
ERRATUM
}

\section{NFкB1 (p50)-deficient mice are not susceptible to multiple low-dose streptozotocin-induced diabetes}

\author{
J G Mabley, G Haskó, L Liaudet, F Soriano, G J Southan, A L Salzman and C Szabó
}

The authors and the journal sincerely apologise for an error in the name of one of the authors in this article, published in
2002, vol 173, on pages 457-464. The name of F Soriano should read as F G Soriano. 